\title{
O Provimento de Feedback Explanatório em um Objeto de Aprendizagem Multimodal
}

\author{
Ygor Corrêa, PGIE / UFRGS - y.correa@bol.com.br \\ Denice Aparecida Fontana Nisxota Menegais, PGIE / UFRGS - denice.menegais@gmail.com \\ Flávia Pereira da Silva, PGIE / UFRGS - flaviaps.poa@gmail.com \\ Maria Cristina Biazus, PGIE / UFRGS - cbiazus@ufrgs.br
}

Resumo: O presente artigo descreve o uso de um Objeto de Aprendizagem Multimodal (OAM) sob a ótica de Gagné (2005), como possibilidade de estímulo à aprendizagem e ao aprimoramento do desempenho de aprendizes na aplicação de novos conhecimentos. Para tanto, baseia-se na Teoria de Projeto Instrucional para elencar diretrizes a fim de que o aprendiz se torne um "produtor de sentido", conforme Moreno e Mayer (2007). O estudo foi realizado com aprendizes do $1^{\circ}$ ano do ensino técnico integrado em Informática do Instituto Federal do Rio Grande do Sul (IFRS) - Campus Ibirubá, na disciplina de Linguagem de Programação I, sobre erros de compilação em linguagem C, causados por termos específicos em língua inglesa. Os resultados obtidos, a partir da implementação do OAM, sugerem que o seu uso foi eficaz no esclarecimento das tarefas que lhes causavam dificuldades na interpretação de tais termos. A utilização do provimento de Feedback Explanatório (FE), inserido nas questões avaliativas do OAM, remeteu o processo de aprendizagem a resultados consideráveis, na medida em que com o provimento de Feedback Corretivo (FC) notou-se a recorrência de tais dificuldades. Os resultados obtidos evidenciam a construção de conhecimento via FE em um OAM como andaimento (VYGOSKTY, 1998) do processo de aprendizagem.

Palavras-chave: Ensino e Aprendizagem. Objeto de Aprendizagem Multimodal. Feedback Explanatório.

\section{The Provision of Explanatory Feedback in a Multimodal Learning Object}

Abstract: This article describes the use of a Multimodal Learning Object (MLO) from Gagné's (2005) perspective as a possible stimulus to learning and improving performance of learners in the application of new knowledge. Therefore, this study is based on the Instructional Design Theory to outline guidelines, so that the student becomes a "sensemaker" according to Moreno and Mayer (2007). The study was conducted with students from the first year of technical education integrated to the Federal Informatics Institute of Rio Grande do Sul (IFRS) - Ibirubá Campus, from the discipline of Programming Language I, on compilation errors in C language, caused by specific terms in English. The results from the implementation of the OAM, suggest that its use was effective in the understanding of the tasks that caused the learners difficulties in the interpretation of such terms. The provision of Explanatory Feedback (EF), inserted in the evaluative questions of the $M L O$, referred the learning process to considerable results, meanwhile in the provision of Corrective Feedback (CF) was noted the recurrence of the difficulties. The results show the construction of knowledge via EF in an MLO as scaffolding (VYGOSKTY, 1998) of the learning process.

Keywords: Teaching and Learning. Multimodal Learning Object. Explanatory Feedback. 


\section{Introdução}

Este estudo debruça-se sobre o provimento de Feedback em um Objeto de Aprendizagem Multimodal (OAM), com vistas a propiciar uma aprendizagem significativa (AUSUBEL et al., 1983) via ferramenta tecnológica HotPotatoes. Alicerçado na Teoria de Projeto Instrucional, este estudo, tem por objetivo elencar princípios que constituem as ações do aprendiz, tornando-o um “produtor de sentido”, segundo Moreno e Mayer (2007, p.312). Entende-se que a navegação em um OAM pode potencializar a aprendizagem caracterizando-a como significativa, quando o design de seu conteúdo está baseado em princípios instrucionais, assim como o estilo de Feedback provido pode aprimorar o desempenho do aprendiz quanto à aplicação de novos conhecimentos (CLARIANA et al., 1991; MORY, 2004). Segundo Moreno e Mayer (2007), os princípios instrucionais estão diretamente ligados a eventos, que têm por finalidade a ativação de processos cognitivos correspondentes, desta maneira, este tudo apresenta a elaboração de um OAM. A prática docente, em sala de aula presencial, apontou para necessidade de utilização de um OAM, a fim de, possivelmente, elucidar dificuldades quanto ao domínio de termos em língua inglesa, em nível básico e intermediário (PASHLER et al., 2005), durante a realização de tarefas de programação. A elaboração de um OAM e o estudo do conceito e aplicação de provimento de feedback está diretamente ligado ao fato que de, em geral, era preciso ampliar o provimento e o estilo de feedback durante a realização de tarefas de compilação.

Considerando que Gagné et al. (2005) propõe nove eventos instrucionais em contexto virtual, foi elaborado um OAM intitulado OAM para interpretação de Erros de Compilação, com o objetivo de capacitar aprendizes do $1^{\circ}$ ano do ensino técnico de Informática integrado ao Instituto Federal do Rio Grande do Sul (IFRS) - Campus Ibirubá, em relação à utilização de termos específicos em língua inglesa, decorrentes de linguagem C acerca de erros de compilação, presentes no conteúdo da disciplina de Linguagem de Programação I. O OAM teve, portanto, a função de auxiliar na tarefa de compreensão da dinâmica de compilar erros de programação, assim como possibilitar a visualização de resultados obtidos por meio da navegação no OAM associado ao provimento de Feedback Explanatório (FE). O OAM, desta maneira, pretendia realizar o andaimento (VYGOSKTY, 1998; HARTMAN, 2002) de um estágio de conhecimento para outro mais avançado. Este artigo está divido nas seguintes seções: 2. Fundamentação teórica; 3. Feedback explanatório x Feedback corretivo 4. O estudo realizado: da construção à implementação de um OAM; 5. Resultados Obtidos; e 6. Considerações Finais.

\section{Fundamentação Teórica}

Tendo em vista que a pesquisa prevê a elaboração e implementação de um OAM, Sweller (2003), referindo-se ao processo de informação ao qual o aprendiz é exposto, afirma que a aprendizagem ocorre de forma mais significativa quando o processo cognitivo humano está alinhado. Nesta concepção, a proporção de informações oferecidas ao aprendiz deve ser compatível com o seu estágio de compreensão. Neste tocante, a Teoria da Carga Cognitiva ressalta que o aprendiz está impossibilitado, dado sua natureza, de processar e absorver diversas informações em sua memória de trabalho, simultaneamente. Essa preocupação faz com que materiais multimídia sejam desenvolvidos com o objetivo de tornar a abordagem do conteúdo mais atraente. Porém, sem acarretar em uma sobrecarga cognitiva desnecessária frente aos elementos multimídia e multimodais, nesse estudo, em um OAM. Perseguindo a ótica de Sweller (2003), Mayer (2001) elaborou alguns princípios 
subjacentes à concepção de um conteúdo via multimídia, com o objetivo de que a aplicação desses vise à redução da sobrecarga cognitiva, potencializando o processo de aprendizagem.

No que diz respeito à elaboração de materiais com finalidades pedagógicas que objetivam a aprendizagem, entende-se que as postulações de Sweller (2003) e Mayer (2001) devem ser levadas em consideração quanto à composição de atividades educacionais em OAMs. Segundo Gagné et al. (2005), o design ou projeto instrucional é definido como o conjunto de eventos externos (métodos, técnicas e recursos) norteados por concepções de ensino e aprendizagem criadas para apoiar processos internos, de forma impressa ou digital. O projeto instrucional abarca uma sequência composta por nove eventos de instrução que têm uma relação de causa e efeito entre si, promovendo, com isso, uma aprendizagem significativa. Esses nove eventos instrucionais apoiam-se em processos internos de aprendizagem e servem como um guia para que o professor possa planejar as suas aulas e produzir materiais educacionais. O professor pode, a partir desse modelo de design instrucional, de acordo com os objetivos pedagógicos e o nível de aprendizagem desejado, usar os eventos mais relevantes para seus aprendizes. Neste entendimento, concebe-se que a adoção de um evento como prioritário, foca-se na intenção de vislumbrar o desenvolvimento de uma habilidade mais do que outras, visando alcançar seu objetivo relacionado ao conteúdo estudado. Diante do que fora mencionado, são apresentados os nove eventos de instruções propostos por Gagné (2005): 1) obter a atenção; 2) descrever os objetivos; 3) recuperar o conhecimento prévio; 4) apresentar o material instrucional; 5) orientar a aprendizagem; 6) fomentar a tarefa realizada; 7) dar retorno sobre o desempenho; 8) avaliar o desempenho; e 9) aumentar a retenção e a transferência. Uma vez que este estudo considera relevante a abordagem pedagógica, inserida em um OAM e relacionada à aprendizagem, passa-se ao conceito de feedback.

\section{O conceito de Feedback enquanto abordagem pedagógica inserido em um OAM}

Na medida em que esta seção concebe o conceito de Interação Homem Computador (IHC), segundo Lemos (2012, p.1) que afirma que este é uma contínua troca de informações entre o usuário e a máquina, entende-se que esta troca está alicerçada na definição de interatividade, como sendo "uma ação dialógica entre homem e técnica sendo uma atividade tecno-social”. Segundo Padovani e Moura (2008), quanto maior o nível de interatividade, maior será o engajamento do aprendiz inserido em um sistema.

Entendendo que é por meio da interatividade que a aprendizagem ocorre, neste caso, via OAM e que o provimento de feedback é dado, adota-se a partir de Mory (2004, p.745) a definição de feedback como "qualquer comunicação ou procedimento apresentado para informar ao aprendiz a exatidão de uma resposta, usualmente, a uma questão instrucional”. Ainda nesta perspectiva, Gagné (1985) detalha o conceito de feedback pontuando que, uma vez fornecido, esse necessita que o aprendiz realize uma verificação externa, quanto à atividade realizada, desta forma, realizando uma metareflexão, passando possivelmente a reter o conteúdo ao qual fora exposto. Brethowen e Smalley (1998, p.61) seguindo tal perspectiva acrescentam que o provimento de feedback enquanto abordagem pedagógica pode capacitar o aprendiz que o recebe, comunicando-lhe aspectos como "manter o bom desempenho, melhorá-lo e sentir-se bem acerca de seus resultados”. Ressalta-se que o aspecto motivacional é evidenciado em outros estudos, como, por exemplo, Song e Keller (2001). 
Tendo pontuado o entendimento de feedback, acrescenta-se a definição desse segundo Moreno e Mayer (2007) quando desta abordagem pedagógica mediada por um OAM na ótica da teoria do projeto instrucional. As atividades orientadas por meio de tecnologias instrucionais promovem uma aprendizagem significativa (MORENO, 2004), quando os aprendizes são submetidos à interação efetiva com o conteúdo disponibilizado em um ambiente, neste caso, no OAM. Moreno (2005) referindo-se à Teoria da Aprendizagem Cognitiva-Afetiva com Mídia (em inglês CATLM - que baseia-se nas premissas sugeridas pelas áreas da cognição e da motivação), aponta para o fato de que a eficácia do processo de aprendizagem está associada à qualidade do feedback provido em um OAM, em relação ao conhecimento que o aprendiz já possui. Entende-se o princípio de feedback como uma forma complementar de expansão da interatividade entre o aprendiz e o OAM. Esta atividade pode ser pensada a partir da utilização de imagens, animações, áudios, vídeos, avatares, etc. Ressalta-se, contudo, que, a fim de evitar a sobrecarga cognitiva do aprendiz (MORENO e MAYER, 2007), deve-se considerar os conhecimentos prévios que o aprendiz possui para que o processo de exposição a novos conhecimentos não se torne excessivo, impedindo que a carga cognitiva seja nociva à aprendizagem (MORENO, 2004; SWELLER et al., 1998; SWELLER, 1999; PAAS et al., 2003).

Nesta perspectiva, são apresentados os conceitos de Feedback Explanatório (FE) e Feedback Corretivo (FC), (MASON e BRUNING, 1999; MORENO, 2004) tendo em vista as implicações exercidas pela adoção do tipo de provimento de Feedback, enquanto abordagem pedagógica. O Feedback Explanatório (FE) consiste em fornecer ao aprendiz uma explicação baseada em um princípio, justificando se as respostas dadas pelo aprendiz estão corretas ou incorretas. O Feedback Corretivo (FC), por sua vez, apenas informa se as respostas estão objetivamente corretas ou incorretas. Moreno e Mayer (2007) referindo-se a estudos realizados com crianças quanto ao provimento FE e FC evidenciaram que a abordagem pedagógica via $\mathrm{FE}$ apresentou resultados satisfatórios quanto à pontuação de crianças em pós-testes. Por outro lado, as crianças que receberam FC não apresentaram uma pontuação significativa dada a qualidade do feedback provido. Segundo CATLM o princípio de FE reduz o processamento irrelevante na tentativa de encontrar uma explicação significativa quando em seu conhecimento prévio ainda não há nenhum modelo mental estruturado. Na seção seguinte, apresenta-se o que concerne à estruturação do OAM a partir do projeto instrucional sob a ótica dos eventos propostos por Gagné (2005), bem como os recursos utilizados na implementação do OAM. Tendo apresentado a perspectiva teórica adotada, passa-se à apresentação do OAM, desde sua construção à implementação, enquanto caráter empírico deste estudo.

\section{O estudo realizado: da construção à implementação de um OAM}

Na medida em que este estudo se dispõe à discussão dos conceitos de Feedback Corretivo (FC) e Feedback Explanatório (FE), faz-se necessário apresentar as razões desencadeadoras de sua conceitualização. Pois, uma vez tendo identificado o tipo de Feedback provido em sala de aula (Figura 1), como sendo o FC que prevalecia, a fim de não ter de retomar todo conteúdo novamente ou atrapalhar aqueles que realizavam as tarefas de forma mais habilidosa, entendeu-se que o OAM elaborado necessitaria de uma abordagem que, de fato, potencializasse a aprendizagem via contexto virtual via FE. Tanto o FC quanto o FE eram providos em sala de aula, embora prevalecesse, durante as aulas práticas de compilação, o FC. O professor da disciplina disse que o provimento de FC 
durante as aulas práticas devia-se à intenção de não desequilibrar o desempenho daqueles que estavam concentrados nas tarefas. Na Figura 1, pode-se notar que o que o feedback provido, na interação professor versus aluno, enquadra-se na definição de FC, entendido como limitado e não potencializador dos processos de aprendizagem, aos quais deveria abarcar, como, por exemplo, basear-se no provimento de um princípio base para justificar se o processo está certo ou errado.

\begin{tabular}{|c|c|c|}
\hline Situação 1 & Situação 2 & Situação 3 \\
\hline Termo: Before & Termo: Expected & Termo: Missing \\
\hline $\begin{array}{l}\text { Aluno A: "-Professor, eu } \\
\text { nåo sei o que significa } \\
\text { before, enta nåo consigo } \\
\text { continuar!" }\end{array}$ & $\begin{array}{l}\text { Aluno B: "-Eu estou com a } \\
\text { mesma dificuldade da outra } \\
\text { aula o que quer dizer } \\
\text { expected?" }\end{array}$ & $\begin{array}{l}\text { Aluno C: "-Tá dando um } \\
\text { erro. Eu acho que nåo sei a } \\
\text { tradução de missing. É } \\
\text { saida?" }\end{array}$ \\
\hline $\begin{array}{l}\text { Professor: "-Para continuar } \\
\text { você deve considerar que } \\
\text { esse termo quer dizer } \\
\text { antes." }\end{array}$ & $\begin{array}{l}\text { Professor: "-Quer dizer } \\
\text { esperado. Lembra?" }\end{array}$ & $\begin{array}{l}\text { Professor: "-Não! Isso } \\
\text { quer dizer ausente ou } \\
\text { faltando." }\end{array}$ \\
\hline $\begin{array}{l}\text { Aluno A: "Ok. Agora deu } \\
\text { certo!" }\end{array}$ & $\begin{array}{l}\text { Aluno B: "-Por isso que eu } \\
\text { não conseguia. Obrigada!" }\end{array}$ & $\begin{array}{l}\text { Aluno C: "-Preciso estudar } \\
\text { esses temos para não } \\
\text { acontecer isso." }\end{array}$ \\
\hline
\end{tabular}

Figura 1 - Provimento de Feedback Corretivo (FC): interação professor x aluno.

A partir das razões expressas para a realização deste estudo, passa-se ao detalhamento do processo de construção do OAM. No processo de construção do $O A M$ para interpretação de Erros de Compilação até sua implementação, partiu-se da definição do modelo conceitual (Classificação dos Termos em Língua Inglesa) que descreve os elementos e as relações relevantes para o objeto em questão, passando para o modelo navegacional acerca de sua interatividade. O modelo conceitual do OAM é constituído pelos conceitos de frase - um exemplo de frase onde um termo é aplicado; fase - uma forma de agrupar os termos; pergunta - uma questão pertencente a uma avaliação para testar os conhecimentos dos aprendizes sobre os termos; e termo - uma palavra que ocorre em algum erro de compilação. A forma como a estrutura do OAM foi criada para armazenar os dados levou em consideração um texto/frase em Inglês, em Português, um áudio deste termo em Inglês e uma imagem da frase onde o termo em Inglês é utilizado.

A fim de definir os perfis para a modelagem navegacional, os 26 alunos da disciplina de Linguagem de Programação I, com faixa etária entre 14 e 16 anos, foram submetidos a um questionário composto por 33 termos em língua inglesa, oriundos de Erros de Compilação recorrentes na disciplina. Em se tratando da abordagem metodológica, elaborou-se uma legenda com as seguintes possibilidades de resposta, quanto aos termos: (i) não reconhecia; (ii) reconhecia e não recordava seu significado; ou (iii) reconhecia plenamente seu significado. Com base nas respostas obtidas, os termos foram divididos em três grupos de acordo com a legenda.

As fases navegacionais foram definidas a partir dos dados resultantes do questionário, permitindo a navegação do aluno pelas fases do OAM com um nível crescente de dificuldade de aplicação dos termos. Nesta fase inicial, foram definidas como: Fase 1reconheciam e sabiam o significado (item iii); Fase 2- reconheciam e não sabiam o significado (item ii); e Fase 3- desconheciam o significado (item i). Dessa maneira, para o modelo navegacional definiu-se o Perfil Básico como aquele em que o aprendiz possuía conhecimento limitado para interpretar os erros apresentados pelo compilador abrangendo as Fases 1 e 2. O Perfil Intermediário definiu-se como sendo aquele em que o aprendiz já possuía conhecimento básico para interpretar os erros, sendo submetido posteriormente aos termos pertencentes à Fase 3. Para avançar de um perfil para o outro, o aprendiz deveria obter aprovação com percentual mínimo de acertos nas questões avaliativas do OAM; caso contrário, o aprendiz deveria navegar novamente na mesma fase e refazer a avaliação. Esta 
abordagem visava, especificamente, a geração de um contexto interativo que provesse feedback explanatório, entendido, como mencionado na seção anterior, como potencializador dos processos de aprendizagem.

Após ter identificado que o FC não supria a demanda gerada em sala de aula, em relação às tarefas de compilação de erros de programação, optou-se por realizar uma abordagem pedagógica em contexto digital, que fosse embasada no provimento de $\mathrm{FE}$. Na Figura 2, apresenta-se um exemplo provimento de FE na interatividade aluno versus OAM. Neste exemplo, ressalta-se, contudo, enquanto conteúdo inserido em uma questão HotPotatoes, quando da visualização apresentada em HTML, o aluno além de saber se sua resposta está certa ou errada, esse recebe um explicação acerca do assunto, tendo por princípio base as regras gramaticais da língua inglesa, uma aplicação em frase e também um link URL para correlacionar o que aprendera durante a navegação pelo OAM.

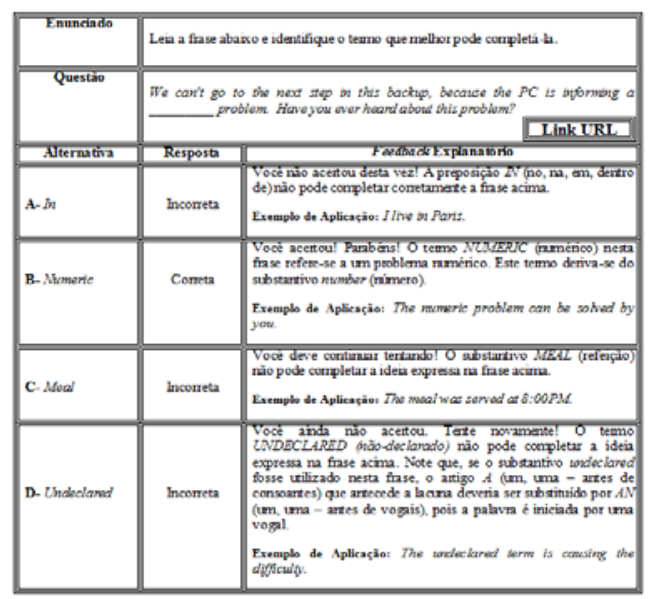

Figura 2 - Provimento de Feedback Explanatório (FE): interatividade OAM x aluno.

Para manter o contexto, a navegação entre os termos de uma mesma frase era guiada e indexada, facultando ao aluno a escolha da ordem de visualização dos termos. O sistema escolhia automaticamente frases que possuíam o termo selecionado; contudo, o OAM recomendava termos não escolhidos pelo aluno para que o mesmo os explorasse após transcorrido certo tempo de navegação por meio de um conjunto de a) tradução; b) áudio; c) imagem e exemplos de aplicações em frases; e d) um link para a exploração em uma frase em língua inglesa. A partir da definição dos perfis, utilizaram-se ferramentas e tecnologias livres de propriedade para desenvolver o OAM, a fim de que o mesmo pudesse ser executado tanto no sistema operacional Linux quanto no sistema operacional Windows, da mesma maneira que foi projetado para utilização tanto em um computador quanto em uma lousa digital. Para tanto, as principais ferramentas utilizadas foram HTML 5.0, CSS, JavaScript, Popcorn, Voki e HotPotatoes. Com a ferramenta HotPotatoes (http://hotpot.uvic.ca), foram elaboradas questões avaliativas com a função de prover feedback explanatório.

A interface inicial do OAM possui um vídeo com um avatar customizado utilizando o Voki, cujo conteúdo foi elaborado e narrado pelo professor da disciplina mencionada, sob orientação, a fim de instigar e motivar os aprendizes. Após assistir o vídeo introdutório, situado na primeira interface, o aprendiz iniciava a navegação pelo Perfil Básico, com a possibilidade de mais de um modo de interação, a saber, pelas modalidades verbal e nãoverbal. Em ambos os perfis descritos, o layout dos vídeos é mantido como padrão. Após a interface em que o aprendiz visualizava um vídeo com um erro de compilação para 
solucioná-lo, uma questão avaliativa gerada pela ferramenta HotPotatoes demonstrava a aplicação do termo contextualizado, a fim de orientar a aprendizagem. Assim, podendo recuperar conhecimentos previamente abordados no OAM, o aprendiz recebia feedback (MAYER, 2001) elaborado previamente pelo professor de acordo com o percentual de acertos e fomentava a tarefa quanto à aplicação do termo e o retorno ao aprendiz em relação a seu desempenho. Como conteúdo complementar, utilizaram-se links de páginas da Web que tratassem de conteúdos relacionados.

Dessa maneira, como forma de aumentar a retenção e a transferência do conteúdo navegado, permitiu-se ao aprendiz que retomasse o conteúdo abordado em situações diversas. Então, por meio dos links, o aprendiz poderia sentir-se instigado a refletir sobre a aplicação dos termos em outros contextos, e não meramente quanto ao ato de interpretação de erros compilação. Ao clicar nestes links, o aprendiz era redirecionado para uma navegação autônoma como forma de construção de conhecimento em um contexto de cibercultura (LÉVY, 1999). Uma vez tendo apresentado a estrutura composicional do OAM elaborado para implementação na disciplina de Linguagem de Programação I, passa-se aos resultados obtidos, como forma de verificar o processo de aprendizagem embasado nos pressupostos teórico-metodológicos adotados.

\section{Resultados Obtidos}

Nesta seção são apresentados os resultados obtidos a partir da implementação do OAM elaborado para a utilização na disciplina de Linguagem Programação I, visando elucidar as dificuldades quando da aprendizagem do procedimento de interpretação de erros de compilação.

Uma vez que a prática de sala de aula evidenciou que, durante a realização de tarefas de compilação, fora necessário, muitas vezes, parar a execução das tarefas para que o professor retomasse o entendimento acerca dos termos em língua inglesa. Este aspecto, conforme relatado pelo professor da disciplina, fazia com que as tarefas fossem realizadas em um período de tempo mais longo do que previsto, assim entendeu-se que, embora o professor provesse FC e FE era preciso ampliar a proporção de FE. Deste modo, o professor em inúmeras situações relatou ter de retomar o conteúdo pertencente à teoria ao invés de prosseguir com prática de compilação. O que se que dizer é que a dinâmica da aula retrocedia ao invés de avançar. Diante da identificação das dificuldades relacionadas, optou-se pela inserção do OAM com vistas a ampliar o provimento de Feedback Explanatório (FE), sendo que o FC, no momento de realização das tarefas parecia prevalecer. A adoção do OAM, então visou qualificar o feedback dado.

No que se refere à metodologia desta pesquisa, inicialmente, fora composta por 26 aprendizes, sendo que, no entanto, apenas 14 participaram da validação do OAM. Deste modo, a turma foi dividida em dois grupos, intitulados aprendizes COM OAM e SEM OAM (ver Figura 3), com 7 aprendizes cada. Quanto à divisão realizada, adotou-se a seguinte abordagem pedagógica: enquanto o grupo de aprendizes COM OAM era exposto ao conteúdo do OAM e recebia FE no laboratório de informática, o grupo SEM OAM realizava as mesmas tarefas de compilação em seus notebooks, em sala de aula, sendo que durante a realização dessas tarefas, o professor tinha como função prover FE.

Os dois grupos foram compostos por aprendizes de diferentes níveis de domínio do conteúdo abordado na disciplina, uma vez considerado o princípio das diferenças individuais, enquanto grupo heterogêneo. 


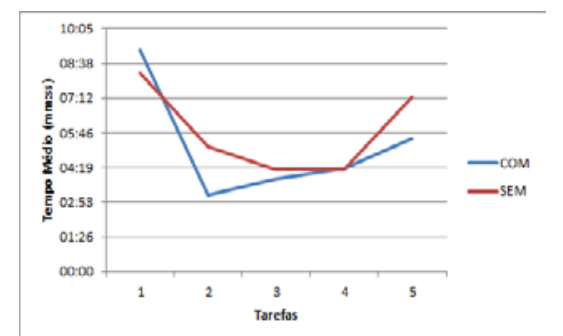

Figura 3 - Resultados a partir da implementação do OAM.

Os sete aprendizes COM OAM navegaram no OAM que fora construído com o propósito de elucidar as dificuldades associadas aos termos em língua inglesa. Após a navegação os aprendizes COM OAM integraram-se aos SEM OAM, sendo estes, submetidos à realização de 5 tarefas, as quais envolviam programas para codificação na linguagem C. O tempo máximo de realização para cada tarefa foi limitado como sendo de 10 minutos, as que não fossem realizadas durante esse período deveriam ser abandonadas. Os resultados da implementação são apresentados no gráfico (Figura 3), percebeu-se que na maioria das tarefas, o tempo médio para realização foi inferior para o grupo de aprendizes que utilizou o OAM. Foi possível notar que, na primeira tarefa, os aprendizes que utilizaram o OAM antes da prática de compilação apresentaram um período de tempo semelhante aos que não utilizaram, talvez isso se deva ao fato de que tenha havido uma expectativa quanto a uma realização satisfatória das tarefas.

Nas tarefas 2, 3 e 5, o grupo COM OAM obteve um tempo relativamente inferior, isso porque os aprendizes ao aprimorar seus conhecimentos em língua inglesa por meio do OAM, conseguiram corrigir os erros de compilação de forma mais ágil. Observa-se que a partir da tarefa 2 e 3 a diferença de tempo foi diminuindo em relação aos aprendizes SEM OAM. Por outro lado, na medida em que as tarefas 4 e 5 tornaram-se mais complexas quanto ao ato de programar, notou-se que o tempo necessário para sua realização tanto para os aprendizes COM OAM, quanto para os aprendizes SEM OAM aumentou. Ressalta-se que o grupo SEM OAM deixou de realizar 12 tarefas, enquanto o grupo COM OAM deixou de realizar apenas 8, não tendo sido estas consideradas na Figura 3. A partir dos resultados obtidos com a implementação do OAM, a partir dos Princípios Multimídia de Mayer (2001), considerou-se os resultados como significativos quando de sua aplicação.

\section{Considerações Finais}

Levando em consideração os resultados obtidos a partir da implementação do OAM elaborado, quanto à base teórica deste estudo, considera-se que os mecanismos de navegação e orientação concebidos a partir da dificuldade inicial de interpretação dos erros de compilação em língua inglesa foram eficazes. Uma vez que, a abordagem pedagógica quanto às tarefas realizadas em sala de aula residia no provimento de feedback corretivo (FC), notou-se que, embora este tipo de feedback fosse oferecido, as dificuldades apresentadas eram reincidentes. Os aprendizes expostos ao FC pareciam não memorizar os termos que constituíam as tarefas. Talvez a não absorção deste conhecimento em uma língua estrangeira deva-se ao fato de que o FC apenas elucidava, momentaneamente, a dificuldade apresentada. Desta maneira, a não contextualização da aplicação dos termos para além da compilação de erros pareceu não fomentar sua retenção.

Em relação ao FC, o feedback explanatório (FE) inserido nas questões avaliativas elaboradas via ferramenta HotPotatoes permitiu a retenção de conhecimentos novos, via 
navegação no OAM. O desempenho dos aprendizes quando avaliados no laboratório de informática, evidenciou que as tarefas que lhes causavam dificuldades de interpretação dos termos, foram em parte elucidadas. A eficácia do FE deve-se ao fato de que este estilo de abordagem pedagógica explica a dificuldade apresentada pelo aprendiz contextualizando-a, de modo que esse possa aplicá-la em outros contextos. Outro fator relevante na constituição do OAM foi o layout da interface, onde o conteúdo foi apresentado de maneira multimodal e multimídia, percebeu-se que este fator não gerou sobrecarga cognitiva, contribuindo assim para a memorização dos termos apresentados.

Em consequência a esta perspectiva, o fator memorização do conteúdo evidenciou que a realização das tarefas executadas pelos aprendizes COM OAM consistiu em um período de tempo inferior aos aprendizes SEM OAM, quase que na totalidade das tarefas. No enquadre da utilização do OAM em sala de aula para fins de aprimoramento de um conteúdo, pode-se dizer que este solucionou parte das dificuldades no que se refere à compilação de erros de programação. Ressalta-se que, além do tempo inferior apresentado pelos aprendizes COM OAM, o desempenho em sala de aula foi satisfatório quanto ao grau de acerto das tarefas. A constituição multimídia e multimodal do OAM contribuiu para o enriquecimento das possibilidades de construção de um feedback que fosse além da definição de certo e errado. Na perspectiva do conceito de Zona de Desenvolvimento Proximal (ZDP), segundo Vygoskty (1998), entende-se que o FE pode acarretar no andaimento de um nível de conhecimento para outro mais complexo, propiciando que a instrução fornecida sirva como andaime (HARTMAN, 2002) para sua progressão cognitiva.

Os resultados obtidos evidenciaram que a utilização de um OAM e o provimento de FE podem auxiliar, significativamente, na aprendizagem de conhecimentos que são entendidos como subsunçores (AUSUBEL et al., 1983), para a absorção de novos conhecimentos, neste caso, o domínio dos termos em língua inglesa com o objetivo de interpretar erros de compilação. Portanto, a análise de dados evidenciou que o OAM propiciou a construção de conhecimento por meio da abordagem pedagógica adotada, neste instrumento, e não a mera aquisição de informação como propõe Mayer (2001).

\section{Referências}

AUSUBEL, D. P.; NOVAK, J. D.; HANESIAN, H. Psicologia educativa: um punto de vista cognoscitivo. 2 ed. México: Trillas, 1983.

AZEVEDO, R., \& Bernard, R. M. A meta-analysis of the effects of feedback in computer-based instruction. Journal of Educational Computing Research, 13(2), 111127, 1995.

CLARIANA, R. B., ROSS, S. M., MORRISON, G. R. The effects of different feedback strategies using computer-administered multiple-choice questions as instruction. Educational Technology Research and Development, 39, 5-17, 1991.

GAGNÉ, Robert et al. Principles of instructional design. 5 ed. Thomson, 2005.

GAGNÉ, R. M. The conditions of learning and the theory of instruction. 4 ed. New York: Holt, Rinehart, and Winston, 1985.

HARTMAN, H. Human learning and instruction New York: City College of City University of New York, 2002. p.23-69. 
LEMOS, André. Anjos interativos e retribalização do mundo: sobre interatividade e interafaces digitais. Disponível em: <http://www.facom.ufba.br/ciberpesquisa/lemos/interativo.pdf>. Acesso: 25 outubro. 2012. LÉVY, Pierre. Cibercultura. São Paulo: Editora 34, 1999.

MASON, B. J., BRUNING, R. Providing feedback in computer-based instruction: What the research tells us. Retrieved February, 15, 2007. Disponível em: <http://dwb4.unl.edu/dwb/Research/MB/MasonBruning.html>. Acesso: 23 outubro. 2012. MAYER, Richard. Multimedia learning. New York: Cambridge University Press, 2001. MAYER, R. E. Cognitive theory of multimedia learning. In R. Mayer (Ed.), Cambridge handbook of multimedia learning. New York: Cambridge University Press, 2005. p. 31-48. MORENO, R, MAYER, R. Interactive Multimodal Learning Environments - Special Issue on Interactive Learning Environments: Contemporary Issues and Trends. Educational Psychology Review, 19, 2007. p.309-326.

MORENO, R. Decreasing cognitive load for novice students: Effects of explanatory versus corrective feedback in discovery-based multimedia. Instructional Science 32. 2004. p.99-103.

MORENO, R. Multimedia learning with animated pedagogical agents. In R. Mayer (Ed.), Cambridge handbook of multimedia learning. New York: Cambridge University Press, 2005. p. 507-524.

MORY, E. H. Feedback Research Revisited. In Jonassen, David H. (Ed), Handbook of Research on Educational Communications and Technology. Mahwah, NJ, US: Lawrence Erlbaum Associates Publishers, 2 ed., 2004. p. 745-783.

PAAS, F., RENKL, A., SWELLER, J. Cognitive load theory and instructional design: Recent developments. Educational Psychologist, 38, 2003. p.1-4.

PASHLER, H., CEPEDA, N. J., WIXTED, J. T., ROHRER, D. When does feedback facilitate learning of words? Journal of Experimental Psychology: Learning, Memory, and Cognition, 31, 2005. p.3-8.

PRIDEMORE, D. R; Klein, J. D. Control of practice and level of feedback in computerbased instruction. Contemporary Educational Psychology, 20, 1995. p.444-450.

SONG, S. H.; KELLER, J. M. Effectiveness of motivationally adaptive computer-assisted instruction on the dynamic aspects of motivation. Educational Technology Research and Development, 49, 2001. p.5-22.

SWELLER, John. Cognitive load theory: a special issue of educational psychologist. LEA, Inc, 2003.

SWELLER, J.; VAN MERRIËNBOER, J.; PAAS, F. Cognitive architecture and instructional design. Educational Psychology Review, 10, 1998. p. 251-296.

SWELLER, J. Instructional design in technical areas. Camberwell, Australia: ACER Press, 1999.

VYGOTSKY, L. S. A formação social da mente: o desenvolvimento dos processos psicológicos superiores. 6 ed. São Paulo: Martins Fontes, 1998. 